\title{
INFIX CONGRUENCES ON A FREE MONOID
}

\author{
C. M. REIS
}

\begin{abstract}
A congruence $\rho$ on a free monoid $X^{*}$ is said to be infix if each class $C$ of $\rho$ satisfies $u \in C$ and $x u y \in C$ imply $x y=1$.

The main purpose of this paper is a characterization of commutative maximal infix congruences. These turn out to be congruences induced by homomorphisms $\tau$ from $X^{*}$ to $\mathbf{N}^{0}$, the monoid of nonnegative integers under addition, with $\tau^{-1}(0)=1$.
\end{abstract}

\section{INTRODUCTION}

Let $X$ be a finite alphabet and $X^{*}$ the free monoid on $X$. A subset $T$ of $X^{*}$ is an infix code if $u$ and $x u y$ in $T$ imply $x y=1$. A congruence $\rho$ on $X^{*}$ is said to be infix (resp. $f$-disjunctive) if each $\rho$-class is an infix code (resp. a finite infix code). $f$-disjunctive congruences, which form a subset of the set of infix congruences, were introduced in [6] and it is, in part, the purpose of this paper to further study these congruences within the broader context of infix congruences. In addition, results analogous to those obtained in [6] for $f$-disjunctive congruences will be proved for infix congruences.

In $\S 2$ we prove some general results on infix congruences. In particular we show that the class of infix congruences is strictly larger than the class of $f$ disjunctive congruences. We also prove that commutative infix congruences are in fact $f$-disjunctive and that commutative maximal infix congruences are cancellative.

A congruence $\rho$ on $X^{*}=\left\{a_{1}, a_{2}, \ldots, a_{n}\right\}^{*}$ is said to be $p$-linear if there exist positive integers $l_{1}, l_{2}, \ldots, l_{n}$ such that $u \equiv v(\rho)$ if and only if $\sum_{i=1}^{n} l_{i}|u|_{a_{i}}$ $=\sum_{i=1}^{n} l_{i}|v|_{a_{i}}$, where $|w|_{a_{i}}$ denotes the number of occurrences of the letter $a_{i}$ in the word $w . \S 3$ is devoted to the characterization of commutative maximal infix congruences. These turn out to be precisely the $p$-linear congruences.

Throughout this paper, $\mathbf{R}$ will denote the real numbers, $\mathbf{Z}$ the integers and $\mathbf{N}$ the natural numbers. The length of a word $w \in X^{*}$ will be denoted by $|w|$ while the syntactic congruence of a language $L$ over $X^{*}$ will be denoted by $P_{L}$. Recall that $P_{L}$ is defined by $u \equiv v\left(P_{L}\right)$ if, for all $x, y \in X^{*}, x u y \in L$ if and only if $x v y \in L$. A congruence $\rho$ will be said to be principal if $\rho=P_{L}$

Received by the editors December 1, 1987.

1980 Mathematics Subject Classification (1985 Revision). Primary 20M05.

Key words and phrases. Infix congruences, free monoid, commutative maximal infix congruences. 
for some language $L$. If $f: M \rightarrow T$ is a monoid homomorphism, then $\operatorname{ker} f$ is the congruence defined by $u \equiv v(\operatorname{ker} f)$ if $f(u)=f(v)$.

As a general reference we recommend G. Lallement's book [5].

\section{GeNeral RESUltS ON INFIX CONGRUENCES}

In [6], it was shown that, given an $f$-disjunctive congruence $\rho$, there exists a principal $f$-disjunctive congruence $P_{L}$ with $\rho \leq P_{L}$. Here we prove a somewhat weaker result for infix congruences. Although the proof is similar, we include it for the sake of completeness. We begin with a lemma.

Lemma 2.1. Let $\rho$ be an infix congruence on $X^{*}$. Then any nontrivial submonoid $M$ of $X^{*}$ meets infinitely many $\rho$-classes.

Proof. Suppose $M$ meets only finitely many $\rho$-classes, say $\bar{w}_{1}, \bar{w}_{2}, \ldots, \bar{w}_{n}$ where $\bar{w}_{i}$ is the $\rho$-class of the word $w_{i} \in M$. Then $\left\{\bar{w}_{1}, \bar{w}_{2}, \ldots, \bar{w}_{n}\right\}$ is a submonoid of $X^{*} / \rho$. Hence, $\bar{w}_{j}$, say, is idempotent, whence $w_{j}^{2} \equiv w_{j}(\rho)$. Since $\rho$ is infix, $w_{j}=1$. It follows that for each $\bar{w}_{i}, \bar{w}_{i}^{k}=1$ for some $k$. Hence $w_{i}=1$ for all $i$. Thus $M$ is the trivial monoid, a contradiction.

Definition 2.2. A language $L \subset X^{*}$ is said to be dense if $X^{*} w X^{*} \cap L \neq \varnothing$ for all $w \in X^{*}$.

Theorem 2.3. Let $\rho$ be an infix congruence. Then there exists a dense language $L$ such that $\rho \leq P_{L}$ and the restriction of $\rho$ to $L$ is $P_{L}$.

Proof. Let $C_{1}, C_{2}, \ldots$ be the classes of $\rho$, so numbered that $m\left(C_{i}\right) \leq m\left(C_{j}\right)$ if $i<j$ where $m\left(C_{i}\right)=\min \left\{|w| \mid w \in C_{i}\right\}$. Let $w_{1} \leq w_{2} \leq \cdots$ be a total ordering of $X^{+}$, the free semigroup on $X$, and choose a certain subset of the set of all $\rho$-classes as follows:

Choose $D_{1}=C_{i_{1}}$ such that $X^{*} w_{1} X^{*} \cap C_{i_{1}} \neq \varnothing$ and let $\alpha_{1}=u_{1} w_{1} v_{1} \in D_{1}$. Choose $D_{2}=C_{i_{2}}$ such that $X^{*} \alpha_{1} w_{2} X^{*} \cap C_{i_{2}} \neq \varnothing$ and let $\alpha_{2}=u_{2} \alpha_{1} w_{2} v_{2} \in D_{2}$. Now, by Lemma 2.1, $X^{*} \alpha_{2} w_{3} X^{*}$ meets infinitely many $\rho$-classes; hence we may choose $D_{3}=C_{i_{3}}$ with $X^{*} \alpha_{2} w_{3} X^{*} \cap C_{i_{3}} \neq \varnothing$ and $\left|u_{2} \alpha_{2} w_{2} v_{2}\right|<m\left(D_{3}\right)$. Let $\alpha_{3}=u_{3} \alpha_{2} w_{3} v_{3} \in D_{3}$. Having chosen $D_{j}, 3 \leq j \leq n$ with $\alpha_{j} \in D_{j}, \alpha_{j}=$ $u_{j} \alpha_{j-1} w_{j} v_{j} \in D_{j}$ and $\left|u_{j-1} u_{j-2} \cdots u_{2} \alpha_{j-1} w_{2} v_{2} \cdots w_{j-1} v_{j-1}\right|<m\left(D_{j}\right)$, choose $D_{n+1}=C_{i_{n+1}}$ with $X^{*} \alpha_{n} w_{n+1} X^{*} \cap C_{i_{n+1}} \neq \varnothing$ and $\left|u_{n} u_{n-1} \cdots u_{2} \alpha_{n} w_{2} v_{2} \cdots w_{n} v_{n}\right|$ $<m\left(D_{n+1}\right)$. Again, this can be done since $X^{*} \alpha_{n} w_{n+1} X^{*}$ meets infinitely many $\rho$-classes by Lemma 2.1. Let $\alpha_{n+1}=u_{n+1} \alpha_{n} w_{n+1} v_{n+1} \in D_{n+1}$. Now set $L=\bigcup_{j=1}^{\infty} D_{j}$. Clearly $\rho \leq P_{L}$ since $\rho$ saturates $L$ and $P_{L}$ is the coarsest congruence saturating $L$. We now show that $D_{r} \not \equiv D_{s}\left(P_{L}\right)$ if $r \neq s$. Assuming $r<s$, we have

$$
\alpha_{s}=u_{s} u_{s-1} \cdots u_{r+1} \alpha_{r} w_{r+1} v_{r+1} \cdots w_{s} v_{s} \in L
$$

and thus $\left(u_{s} u_{s-1} \cdots u_{r+1}, w_{r+1} v_{r+1} \cdots w_{s} v_{s}\right)$ is a context of $\alpha_{r}$. Now

$$
\gamma=u_{s} u_{s-1} \cdots u_{r+1} \alpha_{s} w_{r+1} v_{r+1} \cdots w_{s} v_{s} \notin D_{j}
$$


if $j \leq s$ since $\alpha_{j}$ is a proper factor of $\gamma$ for $j \leq s$ and each $D_{j}$ is infix. Moreover, $|\gamma| \leq\left|u_{s} u_{s-1} \cdots u_{2} \alpha_{s} w_{2} v_{2} \ldots w_{s} v_{s}\right|<m\left(D_{t}\right)$ for all $t>s$ by our choice of the $D_{j}$ 's. Hence $\gamma \notin L$ and $D_{r} \not \equiv D_{s}\left(P_{L}\right)$ proving that the $D_{j}$ 's are $P_{L}$-classes. By our construction, it is clear that $L$ is dense.

Remark. This construction of $L$ does not always yield an infix congruence $P_{L}$.

Corollary 2.4. Let $\rho$ be an infix, cancellative congruence on $X^{*}$. Then $\rho$ is principal.

Proof. Let $L$ be as in Theorem 2.3 and let $u \equiv v\left(P_{L}\right)$. Since $L$ is dense, there exist $x, y \in X^{*}$ with $x u y \in L$. But $x u y \equiv x u y(\rho)$ since the restriction of $\rho$ to $L$ is $P_{L}$. Since $\rho$ is cancellative, $u \equiv v(\rho)$, whence $\rho=P_{L}$.

It is tempting to conjecture that every infix congruence is $f$-disjunctive. The following shows that this is not the case.

Example 2.5. Let $w \in X^{+}, w=a_{1}^{m_{1}} a_{2}^{m_{2}} \cdots a_{s}^{m_{s}}$ where, for all $i, m_{i}>0$, $a_{i} \in X$ and $a_{i} \neq a_{i+1}$. Then the skeleton of $w$, denoted $\operatorname{sk}(w)$, is the word $a_{1} a_{2} \cdots a_{s}$. Define $\operatorname{sk}(1)=1$. For $w$ as above, let $I(w)$ denote $a_{1}^{m_{1}}$ and let $F(w)$ denote $a_{s}^{m_{s}}$. Set $I(1)=F(1)=1$. Now define a congruence $\rho$ on $X^{*}$ as follows:

$$
u \equiv v(\rho) \quad \text { if } I(u)=I(v), F(u)=F(v) \text { and } \mathbf{s k}(u)=\mathbf{s k}(v) .
$$

We prove that each $\rho$-class is infix. Let $u \equiv v(\rho)$. If $u=1, \operatorname{sk}(v)=1$ whence $v=1$ and thus $[1]_{\beta}=\{1\}$. If $|\operatorname{sk}(u)|=1$, then $|\operatorname{sk}(v)|=1$ and since $I(u)=I(v)$, it follows that $u=v$. If $|\operatorname{sk}(u)|=2$, since $I(u)=I(v)$ and $F(u)=F(v)$, we have $u=v$. Thus assume $|\operatorname{sk}(u)|>2$. Then

$$
u=b_{1}^{s_{1}} b_{2}^{s_{2}} \cdots b_{n}^{s_{n}}, \quad v=b_{1}^{s_{1}} b_{2}^{t_{2}} \cdots b_{n-1}^{t_{n-1}} b_{n}^{s_{n}},
$$

where all the exponents are positive, the $b$ 's are letters of $X$ and $b_{i} \neq b_{i+1}$ for all $i$. Suppose $u=p v q$. If $p$ contains a letter other than $b_{1}$, the skeleton of $v$ is changed, which is not possible. Hence $p$ is a power of $b_{1}$. But $p$ cannot be a positive power of $b_{1}$ since $I(u)=I(v)$. Thus $p=1$. Similarly, $q=1$. Clearly $\rho$ has infinitely many infinite classes and is therefore infix but not $f$-disjunctive.

As a particular example of the above, let $X=\{a, b\}$. Then the $\rho$-classes are as follows:

(1) Singleton classes: $[1]_{\rho},\left[a^{s}\right]_{\rho},\left[b^{s}\right]_{\rho},\left[a^{s} b^{t}\right]_{\rho},\left[b^{t} a^{s}\right]_{\rho}$ where $s$ and $t$ are positive integers. (2) Classes of the following four types, all infinite:

(i) $\left[a^{i}(b a)^{s} b^{j}\right]_{\rho}, i, j, s$ positive;

(ii) $\left[b^{i}(a b)^{s} a^{j}\right]_{\rho}, i, j, s$ positive;

(iii) $\left[a^{i}(b a)^{s} b a^{j}\right]_{\rho}, i, j$ positive; $s$ nonnegative;

(iv) $\left[b^{i}(a b)^{s} a b^{j}\right]_{\rho}, i, j$ positive; $s$ nonnegative.

We now consider maximal infix congruences and set the stage for a characterization in $\S 3$ of commutative maximal infix congruences. 
Theorem 2.6. Let $\rho$ be an infix congruence of $X^{*}$. Then there exists a congruence $\mu$ containing $\rho$ such that $\mu$ is maximal subject to being infix.

Proof. Let $\mathscr{F}=\{\tau \mid \tau$ is an infix congruence, $\tau \supset \rho\}$ and let $\left\{\tau_{i}\right\}$ be a chain in $\mathscr{F}$. Then $\bigcup \tau_{i}$ is a congruence and if $u \equiv p u q\left(\bigcup \tau_{i}\right)$, then $u \equiv p u q\left(\tau_{i}\right)$ for some $i$, whence $p=q=1$. Hence $\bigcup \tau_{i} \in \mathscr{F}$. By Zorn, $\mathscr{F}$ has a maximal element $\mu$.

Example 2.7. Referring back to Example 2.5 in the case $X=\{a, b\}$ it is easy to show, though tedious, that the congruence $\rho$ is maximal infix. For, suppose that $\mu$ is an infix congruence with $\mu \supsetneq \rho$. There are various cases to consider. We check here only two cases to give the flavour of the other computations necessary to establish the maximality of $\rho$.

Case (i). Suppose $a^{i}(b a)^{s} b^{j} \equiv a^{u}(b a)^{t} b^{v}(\mu), i, j$ and $s$ positive. Then

$$
b a(b a)^{s} b a \equiv b a(b a)^{t} b a(\mu) .
$$

Hence $(b a)^{s+2} \equiv(b a)^{t+2}(\mu)$. Since $\mu$ is infix, $s=t$ and we have

$$
a^{i}(b a)^{s} b^{j} \equiv a^{u}(b a)^{s} b^{v}(\mu) .
$$

Hence $(b a)^{s+1} b^{j} \equiv(b a)^{s+1} b^{v}(\mu)$. Again, since $\mu$ is infix, $j=v$. Similarly $i=u$.

Case (ii). Suppose $a^{i}(b a)^{s} b a^{j} \equiv b^{u}(a b)^{t} a b^{v}(\mu)$ where $i, j, u$ and $v$ are positive, $s$ and $t$ nonnegative.

Then $a b a(b a)^{s} b a b a \equiv a b(a b)^{t} a b a(\mu)$. Hence $(a b)^{s+3} a \equiv(a b)^{t+2} a(\mu)$. Since $\mu$ is infix, $s+3=t+2$. Thus $a^{i}(b a)^{s} b a^{j} \equiv b^{u}(a b)^{s+1} a b^{v}(\mu)$. Therefore

$$
b a(b a)^{s} b a^{j} \equiv b^{u+1}(a b)^{s+1} a b^{v}(\mu)
$$

and thus

$$
b(a b)^{s+1} a^{j} \equiv b^{u+1}(a b)^{s+1} a b^{v}(\mu) .
$$

Hence

$$
b(a b)^{s+1} a b \equiv b^{u+1}(a b)^{s+1} a b^{v+1}(\mu) .
$$

This contradicts the fact that $\mu$ is infix since $u+1$ and $v+1$ are at least 2 .

We remark that the congruence $\rho$ above is not cancellative. For example

$$
a b^{2} a \equiv a b a(\rho) \quad \text { but } b a \not \equiv a(\rho) .
$$

This is in sharp contrast to the situation when the congruence is commutative and maximal subject to being infix.

Theorem 2.8. Let $\mu$ be a commutative congruence maximal subject to being infix. Then $\mu$ is cancellative.

Proof. Define a congruence $\widehat{\mu}$ on $X^{*}$ by $u \equiv v(\widehat{\mu})$ if there exists $w \in X^{*}$ with $w u \equiv w v(\mu)$. Clearly $\mu \leq \widehat{\mu}$ and $\hat{\mu}$ is a congruence which is cancellative. Suppose now that $u \equiv \operatorname{xuy}(\widehat{\mu})$. Then there exists $w \in X^{*}$ with $w u \equiv w x u y(\mu)$. 
Since $\mu$ is commutative, $u w \equiv w u x y(\mu)$, thus proving that $x y=1$ since $\mu$ is infix. Hence $\widehat{\mu}$ is infix, whence $\mu=\widehat{\mu}$.

We now give an example of a class of commutative congruences which are maximal infix. It will be seen in the next section that this class constitutes all commutative maximal infix congruences.

Example 2.9. Let $X=\left\{a_{1}, a_{2}, \ldots, a_{n}\right\}$ and let $l_{1}, l_{2}, \ldots, l_{n}$ be positive integers. Let $|u|_{a_{i}}$ denote the number of occurrences of $a_{i}$ in the word $u$. Define a congruence $\rho$ by $u \equiv v(\rho)$ if $\sum l_{i}|u|_{a_{i}}=\sum l_{i}|v|_{a_{i}} \cdot \rho$ is in fact $f$-disjunctive since the $l_{i}$ are all positive. It is clearly commutative and cancellative. Maximality will be proved in $\S 3$.

Definition 2.10. A congruence of the type described above will be called $p$ linear.

Given two words $u$ and $v$ in $X^{*}$ we may ask under what conditions there exists an infix congruence $\rho$ such that $u \equiv v(\rho)$. Clearly a necessary condition is that neither word be a factor of the other. That this is not sufficient is shown by the following example.

Example 2.11. Let $X=\{a, b\}, u=a b a b a, v=b a b a^{2} b a$ and let $\rho$ be any congruence with $u \equiv v(\rho)$. Thus

$$
\begin{aligned}
& v^{2}=b a b a \underline{a b a b a b a a b a} \\
& \equiv \text { babababaababaaba }(\rho) \\
& \equiv \text { babababababaabaaba }(\rho) \\
& \equiv b a b v^{2} a b a(\rho)
\end{aligned}
$$

showing that $\rho$ is not infix.

At this writing, the author does not know of a necessary and sufficient condition for two words $u$ and $v$ to be congruent modulo an infix congruence. There is however a simple sufficient condition which we prove in Theorem 2.15 below.

Definition 2.12. On $X^{*}$ define the partial order $\leq$ by $u \leq v$ if $u=u_{1} u_{2} \cdots u_{n}$, $v=v_{1} u_{1} v_{2} u_{2} \cdots u_{n} v_{n+1}, u_{i}, v_{i} \in X^{*}$.

This partial order was studied in [3] by Haines. In particular, he proved that any collection of elements in $X^{*}$ which are incomparable with respect to this partial order must be finite.

Definition 2.13 [7]. A subset $T$ of $X^{*}$ is a hypercode if $T$ is a set of incomparable words relative to the partial order $\leq$.

Theorem 2.14. Let $\rho$ be a commutative infix congruence on $X^{*}$. Then each $\rho$-class is a hypercode, whence $\rho$ is $f$-disjunctive.

Proof. If $u_{1} u_{2} \cdots u_{n} \equiv v_{1} u_{1} v_{2} u_{2} \cdots u_{n} v_{n+1}(\rho)$, then

$$
u_{1} u_{2} \cdots u_{n} \equiv u_{1} u_{2} \cdots u_{n} v_{1} v_{2} \cdots v_{n+1}(\rho) \text {. }
$$

Since $\rho$ is infix, $v_{1} v_{2} \cdots v_{n+1}=1$. 
Theorem 2.15. Let $u$ and $v$ be words of $X^{+}, u \neq v$. Then there exists $a$ commutative, cancellative infix congruence with $u \equiv v(\rho)$ if and only if either $|u|=|v|$ or there exist letters $a_{s}, a_{t} \in X$ with $|u|_{a_{s}}>|v|_{a_{s}}$ and $|u|_{a_{t}}<|v|_{a_{t}}$.

Proof. Suppose $\rho$ is a commutative, cancellative infix congruence with $u \equiv$ $v(\rho)$ and suppose. $|u| \neq|v|$ with $|u|>|v|$, say. Let $|u|_{a_{i}}=x_{i},|v|_{a_{i}}=y_{i}$ for all $i$. Then

$$
a_{1}^{x_{1}} a_{2}^{x_{2}} \cdots a_{n}^{x_{n}} \equiv a_{1}^{y_{1}} a_{2}^{y_{2}} \cdots a_{n}^{y_{n}}(\rho) .
$$

Since $\sum x_{i}>\sum y_{i}$, there exists $s$ with $x_{s}>y_{s}$. If $x_{i} \geq y_{i}$ for all $i$, by cancellativity we would have

$$
a_{1}^{x_{1}-y_{1}} a_{2}^{x_{2}-y_{2}} \cdots a_{s}^{x_{s}-y_{s}} \cdots a_{n}^{x_{n}-y_{n}} \equiv 1(\rho),
$$

contradicting infixity of $\rho$. Hence there exists $t$ with $x_{t}<y_{t}$. Conversely, if $|u|=|v|$, then the length congruence $\lambda$ defined by $w \equiv x(\lambda)$ if $|w|=|x|$ will do. Suppose now $|u| \neq|v|$ and $|u|_{a_{s}}>|v|_{a_{s}},|u|_{a_{t}} \leq|v|_{a_{t}}$. Again, letting $|u|_{a_{i}}=x_{i},|v|_{a_{i}}=y_{i}$, let $S=\left\{i \mid x_{i}-y_{i} \geq 0\right\}$ and let $T=\left\{i \mid x_{i}-y_{i}<0\right\}$. By hypothesis $S \neq \varnothing \neq T$. Consider the nontrivial words $\prod_{i \in S} a_{i}^{x_{i}-y_{i}}$ and $\prod_{i \in T} a_{i}^{y_{i}-x_{i}}$ (the order in which the $a_{i}$ appear is immaterial). Let $x_{i}-y_{i}=s_{i}$, for all $i \in S$, and let $y_{i}-x_{i}=t_{i}$ for all $i \in T$. Let $l_{1}, l_{2}, \ldots, l_{n}$ be positive integers satisfying $\sum_{i \in S} l_{i} s_{i}=\sum_{i \in T} l_{i} t_{i}$. Then for some permutation $\pi$ of $1,2, \ldots, n$ the $p$-linear congruence $\rho$ defined by $w \equiv x(\rho)$ if $\sum l_{\pi(i)}|w|_{a_{i}}=$ $\sum l_{\pi(i)}|x|_{a_{i}}$ is the required congruence identifying $u$ and $v$.

\section{A CHARACTERIZATION OF COMMUTATIVE, MAXIMAL INFIX CONGRUENCES}

It is the purpose of this section to prove that the commutative maximal infix congruences are precisely the $p$-linear congruences defined in 2.10 . We begin by proving the easier half of this result.

Theorem 3.1. Every p-linear congruence $\rho$ of $X^{*}$ is maximal infix.

Proof. Let $X=\left\{a_{1}, a_{2}, \ldots, a_{n}\right\}$ and let $l_{1}, l_{2}, \ldots, l_{n}$ be $n$ positive integers. Let $\rho$ be the $p$-linear congruence defined by $u \equiv v(\rho)$ if $\sum l_{i}|u|_{a_{i}}=\sum l_{i}|v|_{a_{i}}$. Let $\mu$ be a maximal infix congruence with $\mu>\rho$. By Theorem $2.8, \mu$ is cancellative. Since $\mu>\rho$, there exist $v, w \in X^{*}$ with $v \equiv w(\mu)$ but $v \not \equiv$ $w(\rho)$. Let $v=a_{1}^{u_{1}} a_{2}^{u_{2}} \cdots a_{n}^{u_{n}}, w=a_{1}^{x_{1}} a_{2}^{x_{2}} \cdots a_{n}^{x_{n}}$. Since $v \not \equiv w(\rho), l_{1} x_{1}+l_{2} x_{2}+$ $\cdots+l_{n} x_{n} \neq l_{1} u_{1}+l_{2} u_{2}+\cdots+l_{n} u_{n}$. Assume w.l.o.g. that $l_{1} x_{1}+l_{2} x_{2}+\cdots+l_{n} x_{n}>$ $l_{1} u_{1}+\cdots+l_{n} u_{n}$. Since $a_{1}^{k l_{2}} \equiv a_{2}^{k l_{1}}(\mu)$ for all positive integers $k$,

$$
a_{1}^{x_{1}+k l_{2}} a_{2}^{x_{2}} \cdots a_{n}^{x_{n}} \equiv a_{1}^{u_{1}} a_{2}^{k l_{1}+u_{2}} a_{3}^{u_{3}} \cdots a_{n}^{u_{n}}(\mu)
$$

for all $k$. We may thus assume w.l.o.g. that $x_{1}>u_{1}$. Similarly, since $a_{2}^{k l_{3}} \equiv$ $a_{3}^{k l_{2}}(\mu)$ for all positive $k$,

$$
a_{1}^{x_{1}} a_{2}^{x_{2}+k l_{3}} a_{3}^{x_{3}} \cdots a_{n}^{x_{n}} \equiv a_{1}^{u_{1}} a_{2}^{u_{2}} a_{3}^{u_{3}+k l_{2}} \cdots a_{n}^{u_{n}}(\mu)
$$


for all $k$. Thus, again, we may assume that $x_{1}>u_{1}, x_{2}>u_{2}$. Continuing in this fashion, we may assume that $x_{i}>u_{i}, i=1,2, \ldots, n-1$. If $x_{n} \geq u_{n}$,

$$
a_{1}^{u_{1}} a_{2}^{u_{2}} \cdots a_{n}^{u_{n}} \cdot a_{1}^{x_{1}-u_{1}} a_{2}^{x_{2}-u_{2}} \cdots a_{n}^{x_{n}-u_{n}} \equiv a_{1}^{u_{1}} a_{2}^{u_{2}} \cdots a_{n}^{u_{n}}(\mu)
$$

contradicting the fact that $\mu$ is infix. Thus assume that $x_{n}<u_{n}$. Since $\mu$ is cancellative we have

$$
a_{1}^{x_{1}-u_{1}} a_{2}^{x_{2}-u_{2}} \cdots a_{n-1}^{x_{n-1}-u_{n-1}} \equiv a_{n}^{u_{n}-x_{n}}(\mu)
$$

where $l_{1}\left(x_{1}-u_{1}\right)+\cdots+l_{n-1}\left(x_{n-1}-u_{n-1}\right)>l_{n}\left(u_{n}-x_{n}\right)$. Let $s_{i}=x_{i}-u_{i}$, $i=1,2, \ldots, n-1, s_{n}=u_{n}-x_{n}$. Then we have

$$
a_{1}^{s_{1}} a_{2}^{s_{2}} \cdots a_{n-1}^{s_{n-1}} \equiv a^{s_{n}}(\mu) \quad \text { with } l_{1} s_{1}+\cdots+l_{n-1} s_{n-1}>l_{n} s_{n} \text {. }
$$

Now

$$
a_{n}^{l_{i} s_{i}} \equiv a_{i}^{l_{n} s_{i}}(\mu), \quad i=1,2, \ldots, n-1 .
$$

Thus

$$
a_{1}^{l_{n} s_{1}} \cdot a_{2}^{l_{n} s_{2}} \cdots a_{n-1}^{l_{n} s_{n-1}} \equiv a_{n}^{l_{1} s_{1}+l_{2} s_{2}+\cdots+l_{n-1} s_{n-1}}(\mu) .
$$

But

$$
a_{1}^{l_{n} s_{1}} a_{2}^{l_{n} s_{2}} \cdots a_{n-1}^{l_{n} s_{n-1}} \equiv a_{n}^{l_{n} s_{n}}(\mu),
$$

whence

$$
a_{n}^{l_{1} s_{1}+l_{2} s_{2}+\cdots+l_{n-1} s_{n-1}} \equiv a_{n}^{l_{n} s_{n}}(\mu) .
$$

But $l_{1} s_{1}+l_{2} s_{2}+\cdots+l_{n-1} s_{n-1}>l_{n} s_{n}$, contradicting the fact that $\mu$ is infix. Hence $\rho$ is maximal infix.

Remark. If $\rho$ is commutative, cancellative and infix, then $\rho$ is not necessarily maximal infix. For example, the congruence $\rho$ defined by $u \equiv v(\rho)$ if $|u|_{a_{i}}=$ $|v|_{a_{i}}$ for all $a_{i} \in X$ is clearly commutative, cancellative and infix but $\rho \leq \lambda, \lambda$ the length congruence.

To prove that every commutative maximal congruence is $p$-linear, we need several lemmas, some involving ideas from linear topological spaces.

We start with the following simple lemma.

Lemma 3.2. Let $\rho$ be a commutative cancellative congruence on $X^{*}$. Then $\rho$ is infix if and only if $[1]_{\rho}=\{1\}$.

Proof. The necessity is clear. Assume that $[1]_{\rho}=\{1\}$. If $u \equiv x u y(\rho)$, then, by commutativity, $u \equiv u x y(\rho)$, whence $x y \equiv 1(\rho)$ by cancellativity. Therefore $x y=1$ and $\rho$ is infix.

We now start proving a series of results which properly belong to functional analysis. We refer the reader to [4] for a more general discussion.

Definition 3.3. (a) Let $\mathbf{R}$ denote the real numbers. A cone $C$ is a subset of $\mathbf{R}^{n}$ such that (i) $C+C \subset C$; (ii) $a C \subset C$ for all nonnegative real numbers $a$.

(b) Let $\alpha \in \mathbf{R}^{n}, \alpha \neq 0$. The set of vectors $\gamma$ such that $\alpha \cdot \gamma \geq 0$ is called a half-space. Here "." denotes the usual dot product. 
Lemma 3.4 [4]. Let $C$ be a cone of $\mathbf{R}^{n}, C \neq \mathbf{R}^{n}$. Then $C$ is contained in a half-space.

Proof. We may assume w.l.o.g. that the interior $C^{0}$ of $C$ is not empty. Choose $\xi \in C^{0}$. If $-\xi \in C$, then $0=\xi+(-\xi) \in C^{0}$ since translation by $-\xi$ is a homeomorphism. Thus an open neighbourhood of 0 is contained in $C$. But since $a C \subset C$ for all nonnegative real numbers $a$, it follows that $C=\mathbf{R}^{n}$, a contradiction. Therefore $-\xi \notin C$. Applying Zorn's Lemma, there exists a cone $M$ maximal subject to $-\xi \notin M$ and $\xi \in M^{0}$. Now let $\alpha \notin M$. Then by the maximality of $M,-\xi \in M+\mathrm{sp}^{+}\{\alpha\}$ where $\mathrm{sp}^{+}\{T\}$ denotes the set of all linear combinations of vectors of $T$ with nonnegative coefficients. Hence $-\xi=\mu+b \alpha, \mu \in M, b>0$. Therefore $-\alpha=(1 / b)(\xi+\mu)$. Since $\xi \in M^{0}$ and both translation by $\mu$ and multiplication by $1 / b$ are homeomorphisms it follows that $-\alpha \in M^{0}$. Thus

$$
\mathbf{R}^{n}=\left(M \cup-M^{0}\right) \cap\left(-M \cup M^{0}\right)=(M \cap(-M)) \cup M^{0} \cup-M^{0} .
$$

We prove that $M \cap(-M), M^{0}$ and $-M^{0}$ are mutually disjoint and that $M \cap$ $(-M)$ is a hyperplane, i.e., a subspace of dimension $n-1$. Let $\alpha \in M \cap-M^{0}$. Then $\alpha \in M$ and $-\alpha \in M^{0}$, showing that $0 \in M^{0}$, whence, as before $M=$ $\mathbf{R}^{n}$, a contradiction. Therefore $M \cap-M^{0}=\varnothing$ and consequently $-M \cap M^{0}=$ $\varnothing$. Hence the three sets $M \cap-M, M^{0}$ and $-M^{0}$ are disjoint. Clearly $M \cap-M$ is a subspace. We show that it is a hyperplane by proving that each $\alpha \in \mathbf{R}^{n}$ is a linear combination of $\xi$ and some element of $M \cap(-M)$. Let $\alpha \in-M^{0}$. Then $\left\{t \mid t \alpha+(1-t) \xi \in M^{0}\right\}$ and $\left\{t \mid t \alpha+(1-t) \xi \in-M^{0}\right\}$ are nonintersecting sets, open in $[0,1]$ and which do not cover $[0,1]$ since $[0,1]$ is connected. Hence there exists $t_{0}$ with $0<t_{0}<1$ such that $t_{0} \alpha+\left(1-t_{0}\right) \xi \notin M^{0} \cup-M^{0}$. Hence $t_{0} \alpha+\left(1-t_{0}\right) \xi \in M \cap(-M)$ since $\mathbf{R}^{n}=M \cap(-M) \cup M^{0} \cup-M^{0}$. Therefore, $\alpha$ is a linear combination of the fixed vector $\xi$ and a vector of $M \cap(-M)$. If $\alpha \in M^{0},-\alpha \in-M^{0}$ and again $-\alpha$, and thus $\alpha$, is a linear combination of $\xi$ and an element of $M \cap-M$. Hence $M \cap-M$ is indeed a hyperplane and $C$ lies in the half-space $M$.

Definition 3.5. Let $\alpha_{1}, \alpha_{2}, \ldots, \alpha_{k}$ be vectors of $\mathbf{R}^{n}$ and let $S$ be a subset of $\mathbf{R}$ containing 0 . We shall say that the set $\left\{\alpha_{1}, \alpha_{2}, \ldots, \alpha_{k}\right\}$ of vectors has property $P$ with respect to $S$ if the following holds: $\sum c_{i} \alpha_{i}=0$ and $c_{i} \in S$ for all $i$ imply $c_{i}=0$ for all $i$.

Corollary 3.6. Let $\left\{\alpha_{1}, \alpha_{2}, \ldots, \alpha_{k}\right\}$ be a set of vectors of $\mathbf{R}^{n}$ having property $P$ with respect to $\mathbf{R}^{+}$, where $\mathbf{R}^{+}$denotes the nonnegative real numbers. Then there exists a nonzero vector $\alpha$ such that $\alpha \cdot \alpha_{i} \geq 0$ for all $i$.

Proof. $C=\operatorname{sp}^{+}\left\{\alpha_{1}, \alpha_{2}, \ldots, \alpha_{k}\right\}$ is a cone. If $\beta$ and $-\beta \in C$, then $\beta=$ $\sum b_{i} \alpha_{i},-\beta=\sum b_{i}^{\prime} \alpha_{i}$, with $b_{i}$ and $b_{i}^{\prime}$ nonnegative. Thus $0=\sum\left(b_{i}+b_{i}^{\prime}\right) \alpha_{i}$ whence $b_{i}+b_{i}^{\prime}=0$ for all $i$. Since $b_{i}$ and $b_{i}^{\prime}$ are nonnegative for all $i$, it 
follows that $b_{i}=b_{i}^{\prime}=0$ for all $i$. Hence, for all $\gamma \in C, \gamma \neq 0,-\gamma \notin$ $C$ showing that $C \neq \mathbf{R}^{n}$. By Lemma 3.4, $C$ is contained in a half-space determined by the hyperplane $\alpha \cdot \xi=0$. By choosing $\alpha$ with the appropriate sign, we may assume $\alpha \cdot \alpha_{i} \geq 0$ for all $i$.

Corollary 3.7. Let $\left\{\alpha_{1}, \alpha_{2}, \ldots, \alpha_{k}\right\}$ be vectors of $\mathbf{R}^{n}$ with property $P$ with respect to $\mathbf{R}^{+}$. Then there exists a vector $\beta$ such that $\beta \cdot \alpha_{i}>0$ for all $i$.

Proof. By the previous result, there exists $\alpha \neq 0$ with $\alpha \cdot \alpha_{i} \geq 0$ for all $i$. Let $\beta$ be a vector with $\beta \cdot \alpha_{i}>0$ for the largest number of $\alpha_{i}$ 's. Let $S$ be the set of those $\alpha_{i}$ with $\beta \cdot \alpha_{i}>0$ and $T$ the set of those $\alpha_{i}$ with $\beta \cdot \alpha_{i}=0$ (note that $S$ could be $\varnothing)$. Let $V=\operatorname{sp}\left\{\alpha_{i} \mid \alpha_{i} \in T\right\}$. Then $\operatorname{dim} V=t \leq n-1$. By the previous result, there exists a hyperplane $H$ of $V$ with $T$ contained in the half-space determined by $H$. Now $H=\widehat{H} \cap V$ where $\widehat{H}$ is a hyperplane of $\mathbf{R}^{k}$ given by $\gamma \cdot \zeta=0$, say. Clearly $T$ lies on one side of $\widehat{H}$ and we may assume $\gamma \cdot \alpha_{i} \geq 0$ for all $\alpha_{i} \in T$. If $\gamma \cdot \alpha_{i}=0$ for all $\alpha_{i} \in T, V=\operatorname{sp}\left\{\alpha_{i} \mid \alpha_{i} \in T\right\} \subset \widehat{H}$, whence $V=\widehat{H} \cap V=H$, a contradiction. Thus $\gamma \cdot \alpha_{i}>0$ for some $\alpha_{i} \in T$. By multiplying $\gamma$ by a suitable positive scalar we may assume $\|\gamma\|<\beta \cdot \alpha_{i} /\left\|\alpha_{i}\right\|$ for all $\alpha_{i} \in S$. (Here, \|\| denotes the usual Euclidean norm). Consider now $\beta+\gamma$. If $\alpha_{i} \in T,(\beta+\gamma) \cdot \alpha_{i}=\beta \cdot \alpha_{i}+\gamma \cdot \alpha_{i}=\gamma \cdot \alpha_{i}$ and if $\alpha_{i} \in S$, $(\beta+\gamma) \cdot \alpha_{i}=\beta \cdot \alpha_{i}+\gamma \cdot \alpha_{i}$. But by the Cauchy-Schwarz inequality,

$$
\left|\gamma \cdot \alpha_{i}\right| \leq\|\gamma\|\left\|\alpha_{i}\right\| \leq \beta \cdot \alpha_{i} /\left\|\alpha_{i}\right\| \cdot\left\|\alpha_{i}\right\|=\beta \cdot \alpha_{i} .
$$

Hence if $\alpha_{i} \in S,(\beta+\gamma) \cdot \alpha_{i}=\beta \cdot \alpha_{i}+\gamma \cdot \alpha_{i}>0$. But for at least one $\alpha_{j} \in T$, $\beta \cdot \alpha_{j}>0$, whence $(\beta+\gamma) \alpha_{j}>0$. The maximality of $S$ is thus contradicted proving that $\beta \cdot \alpha_{i}>0$ for all $i$.

Corollary 3.8. Given vectors $\alpha_{1}, \alpha_{2}, \ldots, \alpha_{k}$ in $\mathbf{Z}^{n}$ with property $P$ with respect to $\mathbf{N}^{0}$, then there exists a vector $\alpha \in \mathbf{Z}^{n}$ such that $\alpha \cdot \alpha_{i}>0$ for all $i$.

Proof. We first need to show that property $P$ with respect to $\mathbf{N}^{0}$ implies property $P$ with respect to $\mathbf{R}^{+}$. Suppose by way of contradiction that this is not the case. We may assume w.l.o.g. that there exist real numbers $r_{i}, i=1,2, \ldots, k$, all nonzero, such that $\sum r_{i} \alpha_{i}=0$. Define a linear transformation $T: \mathbf{R}^{k} \rightarrow$ $\operatorname{sp}\left\{\alpha_{1}, \alpha_{2}, \ldots, \alpha_{k}\right\}$ by $T:\left(c_{1}, c_{2}, \ldots, c_{k}\right) \rightarrow \sum_{i=1}^{k} c_{i} \alpha_{i}$. The kernel of $T$ has a basis $\beta_{1}, \beta_{2}, \ldots, \beta_{s}$ where $\beta_{i} \in \mathbf{Z}^{k}$. Let $\left(r_{1}, r_{2}, \ldots, r_{k}\right)=\sum b_{i} \beta_{i}$ and let $N\left(\left(r_{1}, r_{2}, \ldots, r_{k}\right) ; \delta\right)$ be a neighbourhood of $\left(r_{i}, r_{2}, \ldots, r_{k}\right)$ of radius $\delta>0$ such that $N\left(\left(r_{1}, r_{2}, \ldots, r_{k}\right) ; \delta\right) \subset\left\{\left(x_{1}, x_{2}, \ldots, x_{k}\right) \mid x_{i}>0\right\}$. Choose rationals $p_{i} / q_{i}$ so that $\left|p_{i} / q_{i}-b_{i}\right|<\delta / \sum\left\|\beta_{j}\right\|$ for all $i$. Then

$$
\begin{aligned}
\left\|\left(r_{1}, r_{2}, \ldots, r_{n}\right)-\sum\left(p_{i} / q_{i}\right) \beta_{i}\right\| & =\left\|\sum\left(b_{i}-p_{i} / q_{i}\right) \beta_{i}\right\| \\
& \leq \sum\left|b_{i}-p_{i} / q_{i}\right|\left\|\beta_{i}\right\|<\delta .
\end{aligned}
$$

Hence

$$
\gamma=\sum\left(p_{i} / q_{i}\right) \beta_{i} \in N\left(\left(r_{1}, r_{2}, \ldots, r_{k}\right) ; \delta\right) \subset\left\{\left(x_{1}, x_{2}, \ldots, x_{k}\right) \mid x_{i}>0\right\} .
$$


But $\gamma \in \operatorname{ker} T$ and $\gamma$ has positive rational coordinates. Therefore some positive multiple of $\gamma$, say $\left(c_{1}, c_{2}, \ldots, c_{k}\right)$, has positive integral coordinates. But $\sum c_{i} \alpha_{i}=0$, a contradiction. Therefore $\operatorname{sp}^{+}\left\{\alpha_{1}, \alpha_{2}, \ldots, \alpha_{k}\right\}$ has the property in the hypothesis of Corollary 3.7. Hence, by Corollary 3.7, there exists $\beta \in \mathbf{R}^{n}$ with $\beta \cdot \alpha_{i}>0$ for all $i$. But, by continuity of the dot product, there exists a vector $\alpha^{\prime}$ with rational coordinates such that $\alpha^{\prime} \cdot \alpha_{i}>0$ for all $i$. Multiplying $\alpha^{\prime}$ by a suitable positive integer yields a vector $\alpha \in \mathbf{Z}^{n}$ with $\alpha \cdot \alpha_{i}>0$ for all $i$.

The next theorem is the cornerstone of the proof that each commutative maximal infix congruence is $p$-linear.

Theorem 3.9. Let $M=\left\langle m_{1}, m_{2}, \ldots, m_{k}\right\rangle$ be a finitely generated cancellative, commutative monoid with trivial group of units. Then there exists a homomorphism $\chi: M \rightarrow \mathbf{N}^{0}$ given by

$$
\chi: \prod\left(m_{i}^{c_{i}}\right) \rightarrow \sum_{i=1}^{k} l_{i} c_{i}, \quad l_{i} \text { positive integers. }
$$

Proof. Let $G$ be the group of fractions of $M$. Then $G$ is generated, qua group, by $m_{1}, m_{2}, \ldots, m_{k}$. Thus $G \stackrel{\varphi}{\approx} T \oplus \mathbf{Z}^{n}$ where $k \geq n, T$ is a finite abelian group and $n \geq 1$ since $M$ is aperiodic. Let $\varphi\left(m_{i}\right)=\left(t_{i}, \alpha_{i}\right), i=$ $1,2, \ldots, k$. Suppose that $\sum_{i=1}^{k} c_{i} \alpha_{i}=0, c_{i} \in \mathbf{N}^{0}$ and let $p \in \mathbf{N}$ with $p T=$ (0). Then $\varphi\left(\prod m_{i}^{c_{i} p}\right)=\left(0, \sum p c_{i} \alpha_{i}\right)=(0,0)$. Since $\varphi$ is injective, it follows that $\prod m_{i}^{c_{i} p}=1$. But since the group of units of $M$ is trivial, we have $c_{i} p=0$ for all $i$ and thus $c_{i}=0$ for all $i$. Letting $\pi$ denote the projection of $G$ onto $\mathbf{Z}^{n}$, the mapping $\psi=\left.\pi \varphi\right|_{M}$ is a monoid homomorphism of $M$ to $\mathbf{Z}^{n}$. Let $V=\left\{\sum_{i=1}^{k} c_{i} \alpha_{i} \mid c_{i} \in \mathbf{N}^{0}\right\}$. We now prove that there exists a monoid homomorphism $\tau: V \rightarrow \mathbf{N}^{0}$ given by $\tau\left(\sum_{i=1}^{k} c_{i} \alpha_{i}\right)=\sum_{i=1}^{k} l_{i} c_{i}$ where the $l_{i}$ are positive integers. Since $\left\{\sum_{i=1}^{k} r_{i} \alpha_{i} \mid r_{i} \in \mathbf{R}\right\}=\mathbf{R}^{n}$, we may assume w.l.o.g. that $\left\{\alpha_{1}, \alpha_{2}, \ldots, \alpha_{n}\right\}$ forms a basis. Let $\alpha_{j}=\sum_{i=1}^{n} b_{j i} \alpha_{i}$, for all $j, j \geq n+1$ where the $b_{j i}$ are rational since the $\alpha$ 's are all in $\mathbf{Z}^{n}$. Let $\beta_{j}=\left(b_{j 1}, b_{j 2}, \ldots, b_{j n}\right)$, $j \geq n+1$. We now show that the system of inequalities $\beta_{j} \cdot \xi>0, j \geq$ $n+1$, has a solultion $t=\left(p_{1}, p_{2}, \ldots, p_{n}\right)$ where each $p_{i}$ is a positive integer. Let $\mathscr{E}_{i}, i=1,2, \ldots, n$, be the standard basis of $\mathbf{R}^{n}$ and consider the set $\left\{\mathscr{E}_{1}, \mathscr{E}_{2}, \ldots, \mathscr{E}_{n}, \beta_{n+1}, \ldots, \beta_{k}\right\}$. Let $c_{1} \mathscr{E}_{1}+c_{2} \mathscr{E}_{2}+\cdots+c_{n} \mathscr{E}_{n}+c_{n+1} \beta_{n+1}+\cdots+$ $c_{k} \beta_{k}=0, c_{i} \in \mathbf{N}^{0}$. Then $c_{i}+\sum_{j=n+1}^{k} c_{j} b_{j i}=0$ for $i=1,2, \ldots, n$. Now

$$
\begin{aligned}
\sum_{i=1}^{k} c_{i} \alpha_{i} & =\sum_{i=1}^{n} c_{i} \alpha_{i}+\sum_{j=n+1}^{k} \sum_{i=1}^{n} c_{j} b_{j i} \alpha_{i} \\
& =\sum_{i=1}^{n}\left(c_{i}+\sum_{j=n+1}^{k} c_{j} b_{j i}\right) \alpha_{i}=0 .
\end{aligned}
$$


Hence $c_{i}=0$ for all $i, 1 \leq i \leq k$, since $\left\{\alpha_{1}, \alpha_{2}, \ldots, \alpha_{k}\right\}$ has property $P$ with respect to $\mathbf{N}^{0}$. Thus the set of vectors $\left\{\mathscr{E}_{1}, \mathscr{E}_{2}, \ldots, \mathscr{E}_{n}, \boldsymbol{\beta}_{n+1}, \ldots, \boldsymbol{\beta}_{k}\right\}$ has property $P$ with respect to $\mathbf{N}^{0}$. By Corollary 3.8 , there exists a vector $\mathscr{H}=\left(q_{1}, q_{2}, \ldots, q_{n}\right) \in \mathbf{Z}^{n}$ such that $\mathscr{H} \cdot \mathscr{E}_{i}>0$ for all $i, 1 \leq i \leq k$, and $\mathscr{H} \cdot \beta_{j}>0$ for all $j, n+1 \leq j \leq k$. Since $\mathscr{H} \cdot \mathscr{C}_{i}=q_{i}$, it follows that all the $q_{i}$ are positive integers. Since $\mathscr{H} \cdot \beta_{j}$ is rational for all $j$, an appropriate positive integral multiple, say $\left(p_{1}, p_{2}, \ldots, p_{n}\right)$, of $\mathscr{H}$ is such that $\left(p_{1}, p_{2}, \ldots, p_{n}\right) \cdot \beta_{j}$ is a positive integer for all $j$. Now define a linear transformation $T: \mathbf{R}^{n} \rightarrow \mathbf{R}$ by setting $T\left(\alpha_{i}\right)=p_{i}, i=1,2, \ldots, n$. Then $T\left(\alpha_{j}\right)=\left(p_{1}, p_{2}, \ldots, p_{n}\right) \cdot \beta_{j} \in \mathbf{N}$ for $j \geq n+1$. Hence the restriction $\tau$ of $T$ to $V$ is a monoid homomorphism from $V$ to $\mathbf{N}^{0}$. Let $\chi=\tau \psi$. Then

$$
\chi\left(\pi m_{i}^{c_{i}}\right)=\sum_{i=1}^{n} p_{i} c_{i}+\sum_{j=n+1}^{k}\left[\left(p_{1}, p_{2}, \ldots, p_{n}\right) \cdot \beta_{j}\right] c_{j} .
$$

Hence, setting $l_{i}=p_{i}, i=1,2, \ldots, n$, and $l_{j}=\left(p_{1}, \ldots, p_{n}\right) \cdot \beta_{j}, j \geq n+1$, we have that the $l_{i}$ are positive integers and $\chi\left(\prod m_{i}^{c_{i}}\right)=\sum_{i=1}^{k} l_{i} c_{i}$.

We are now able to prove the result we have been aiming for.

Theorem 3.10. Let $\mu$ be a commutative maximal infix congruence on $X^{*}=\left\{a_{1}, a_{2}, \ldots, a_{n}\right\}^{*}$. Then $\mu$ is p-linear.

Proof. By Theorem 2.8, $X^{*} / \mu$ is cancellative. Also, since $\mu$ is infix, the group of units is trivial. By the preceding theorem, there exists a homomorphism $\chi: X^{*} / \mu \rightarrow \mathbf{N}^{0}$ given by $\chi\left(\Pi \bar{a}_{i}^{c_{i}}\right)=\sum l_{i} c_{i}$ where the $l_{i}$ are positive integers and $\bar{a}_{i}$ is the $\mu$-class of $a_{i}$. Let $\nu: X^{*} \rightarrow X^{*} / \mu$ be the quotient homomorphism. Then $\operatorname{ker} \chi \nu \geq \mu$ and $\operatorname{ker} \chi \nu$ is cancellative. But since $[1]_{\operatorname{ker} \chi \nu}=\{1\}$, by Lemma 3.2, $\operatorname{ker} \chi \nu$ is infix. Hence $\operatorname{ker} \chi \nu=\mu$ by maximality of $\mu$. Thus $a_{1}^{x_{1}} a_{2}^{x_{2}} \cdots a_{n}^{x_{n}} \equiv a_{1}^{u_{1}} a_{2}^{u_{2}} \cdots a_{n}^{u_{n}}(\mu)$ if and only if

i.e., if and only if

$$
\chi \nu\left(a_{1}^{x_{1}} a_{2}^{x_{2}} \cdots a_{n}^{x_{n}}\right)=\chi \nu\left(a_{1}^{u_{1}} a_{2}^{u_{2}} \cdots a_{n}^{u_{n}}\right)
$$

$$
\sum l_{i} x_{i}=\sum l_{i} u_{i} \text {. }
$$

\section{REFERENCES}

1. S. Eilenberg, Automata, languages and machines, vol. A, Academic Press, 1974.

2. Y. Q. Guo, H. J. Shyr and G. Thierrin, $f$-disjunctive languages, Internat. J. Comput. Math. 18 (1986), 219-237.

3. L. H. Haines, On free monoids partially ordered by embedding, J. Combin. Theory 6 (1969), 94-98.

4. J. L. Kelley and I. Namioka, Linear topological spaces, Van Nostrand, Princeton, N.J., 1963.

5. G. Lallement, Semigroups and combinatorial applications, Wiley, 1979.

6. C. M. Reis, A note on $f$-disjunctive languages, Semigroup Forum 36 (1987), 159-165.

7. G. Thierrin and H. J. Shyr, Hypercodes, Inform. and Control 24 (1974), 45-54.

Department of Mathematics, University of Western Ontario, London, Ontario, CANADA 